\title{
Potential Ebola drug targets - filling the gap: a critical step forward towards the design and discovery of potential drugs
}

\author{
Marissa Balmith \& Mahmoud E.S. SolimaN* \\ Molecular Modeling and Drug Design Research Group, School of Health Sciences, University of KwaZulu-Natal, Westville \\ Campus, Durban 4001, South Africa; e-mail: soliman@ukzn.ac.za
}

\begin{abstract}
Among the classified neglected infectious diseases, the Ebola virus (EboV) remains a challenging epidemic. This deadly virus has been reported as a category A bioweapon organism by the World Health Organization due to the serious threat it poses. To date, Ebola drug discovery proves challenging. Proteins need to be targeted at the relevant biologically active site for drug or inhibitor binding to be effective. Due to insufficient experimental data to confirm the biologically active binding site for novel protein targets, researchers often rely on computational prediction methods to identify binding sites. Many computational studies have attempted to identify the biological active site for EboV proteins, however, the methods employed are not sufficiently validated. This has prompted us to provide a comprehensive molecular understanding of the various targets of the EboV, including three-dimensional structures, active site identification and further validation. Herein we report the account of a three-dimensional homology model of the unresolved EboV RNA-dependent RNA polymerase (RdRp), as well as a comprehensive analysis of the binding site residues of all proteins of the EboV. Docking-aided active site determination was carried out to identify possible active sites on the homology model of RdRp. Binding free energy calculations revealed subtle differences in the binding at each site. These results can also provide some potential clues for further design of novel inhibitors to treat this killer virus and is a critical cornerstone of research into the EboV.
\end{abstract}

Key words: Ebola virus; biological targets; computer aided drug design; active site residues; homology modeling.

Abbreviations: 3D, three-dimensional; CADD, computer-aided drug design; CASTp, computed atlas of surface topography of proteins; EboV, Ebola virus; GP, glycoprotein; MD, molecular dynamics; NP, nucleoprotein; PDB, Protein Data Bank; POOL, partial order optimum likelihood; RBD, receptor binding domain; RdRp, RNA-dependent RNA polymerase.

\section{Introduction}

Since the largest Ebola outbreak in 2014, this killer virus has infected 27,621 people and claimed the lives of an estimated 11,268 people globally (Haque et al. 2015; Martínez et al. 2015). Despite extensive ongoing Ebola virus (EboV) research, to date, no cure has been found for this deadly virus. The EboV is a lipid-enveloped, negatively stranded RNA virus affecting both human and non-human primates (Stahelin 2014; MuyembeTamfum et al. 2012). The disease is characterized by severe hemorrhagic fever, focal necrosis of the liver, kidney and spleen, bleeding diathesis and sudden shock with a mortality rate of $90 \%$ (Gebre et al. 2014). Thus far, four species of the virus have been identified; these include Zaire, Sudan, Ivory Coast and Reston EboV with the highest fatality attributed to the Zaire species (Muyembe-Tamfum et al. 2012; Chiappelli et al. 2015).

The EboV genome encodes seven structural proteins, which serve as potential drug targets (Martínez et al. 2015). These drug targets present binding pockets also known as binding sites, which are typically located on the surface concavities of proteins, whereas pockets where smaller drug-like ligands bind can be found in deeper cavities. In the event that a protein function is associated with that of a specific binding site of a virus, such as Ebola, it would be possible to target the binding site, hereby enabling treatment of the virus. This can be achieved through the use of drug-like molecules that halt the progression of the disease by blocking diseaserelated protein biochemical function.

The frequent outbreak of the EboV has drawn much attention to drug development for this deadly pathogen. Several approaches have been employed in the development of drugs to combat Ebola, such as the development of peptides, application of monoclonal antibodies, small molecule inhibitors, recombinant DNA vectors as well as repurposing existing drugs (Haque et al. 2015; Nyakatura et al. 2015). Therapeutics target and attack the virus at different stages of its life cycle, thereby halting virus replication and reducing destruction of the host immune system (Feldmann \& Geisbert 2011). There remains a huge gap in literature with regard to the guidelines towards the discovery of anti-

\footnotetext{
* Corresponding author
} 
Table 1. Ebola virus drug targets. ${ }^{a}$

\begin{tabular}{|c|c|c|c|c|c|}
\hline EboV & PDB code & Structure & Function & Length & Ref. \\
\hline GP & $5 \mathrm{~F} 18$ & & $\begin{array}{l}\text { Binds to receptors on the cell surface and } \\
\text { membrane fusion; pathogenicity }\end{array}$ & 676 & {$[1-3]$} \\
\hline VP40 & $1 \mathrm{H} 2 \mathrm{C}$ & & $\begin{array}{c}\text { Viral assembly and budding; structural } \\
\text { integrity of viral particles; maturation of the } \\
\text { virion }\end{array}$ & 326 & {$[4]$} \\
\hline VP24 & 4M0Q & & $\begin{array}{l}\text { Nucleocapsid formation; encapsulates and } \\
\text { shields viral genome from nucleases; viral } \\
\text { replication }\end{array}$ & 251 & {$[5-7]$} \\
\hline VP30 & $2 \mathrm{I} 8 \mathrm{~B}$ & & Viral transcription activator & 288 & {$[8,9]$} \\
\hline VP35 & 4IJE & & $\begin{array}{l}\text { Multi virulence functionality; innate immune } \\
\text { antagonist and an RNAi silencing suppressor }\end{array}$ & 340 & {$[10,11]$} \\
\hline NP & $4 \mathrm{Z9P}$ & & $\begin{array}{c}\text { Viral replication; scaffold for additional viral } \\
\text { proteins }\end{array}$ & 739 & {$[12-14]$} \\
\hline RdRp & Not available & & Synthesis of positive sense RNA & 2212 & {$[12]$} \\
\hline
\end{tabular}

${ }^{a}$ EboV, Ebola virus proteins; VP40, matrix protein VP40. Structures were retrieved from PDB. Ref., references: [1] Lee et al. 2009; [2] Dahms et al. 2014; [3] Lai et al. 2014; [4] Hartlieb \& Weissenhorn 2006; [5] Noda et al. 2007; [6] Watt et al. 2014; [7] Huang et al. 2002; [8] Mühlberger 2013; [9] Okumura et al. 2010; [10] Haasnoot et al. 2007; [11] Leung et al. 2011; [12] Watanabe et al. 2006; [13] Noda et al. 2011; [14] Johansen et al. 2013.

Ebola inhibitors, which might be due to the lack of information on the Ebola drug targets, binding sites and mechanisms of action. Finding novel, effective drugs at present is heavily dependent on the investigation and understanding of the molecular basis of this pathogenic virus. A lack of experimental data in literature concerning potential Ebola drug targets, their inhibitors, as well as strategies for the design of drugs and the issue of drug resistance has prompted research in this field.

\section{Biological targets of the EboV}

Over the years, increasing interest has been placed on identifying proteins whose biological functions are crucial and can be linked to various severe diseases. These proteins then become biological targets for inhibitors or drug molecules, which could be used in halting or treating these deadly diseases via therapeutic action or by inhibiting the function of the target protein. In order for drugs or inhibitors to be effective, the protein must be targeted at the relevant biological active site. A lack of experimental data to confirm the biological active site of novel protein targets has prompted researchers towards the use of computational tools for the prediction of pos- sible binding sites. This has become a valuable platform for experimental and computational efforts towards the design and discovery of novel drugs and inhibitors that could be used to target these proteins linked to various diseases.

The EboV is a single-stranded, non-segmented, negative RNA genome comprising $\sim 19,000$ nucleotides in length (Wilson et al. 2001). The EboV encodes seven structural proteins, which serve as potential drug targets that is the glycoprotein (GP), matrix protein (VP40), nucleoprotein (NP), nucleocapsid proteins (VP30, VP24 and VP35), and RNA-dependent RNA polymerase (RdRp) (Martínez et al. 2015; Rougeron et al. 2015) (Table 1). Due to the integral role that these proteins play in the life cycle of the virus, EboV proteins are a major target for drug therapy (Picazo \& Giordanetto 2014).

\section{Reported studies on active site identification of EboV targets}

Table 2 highlights the identified and unidentified active site residues of all biological targets of the EboV supported by evidence experimentally or in silico. 
Table 2. Status quo of the available drug targets of the EboV.

\begin{tabular}{|c|c|c|c|}
\hline Targets & Active site residues & PDB code & Ref. \\
\hline GP & Unvalidated experimentally (experimental and in silico methods) & $5 \mathrm{~F} 18$ & {$[1,2]$} \\
\hline VP24 & Unvalidated experimentally (in silico methods) & $4 \mathrm{M} 0 \mathrm{Q}$ & {$[3,4]$} \\
\hline VP30 & Unvalidated experimentally (in silico methods) & $2 \mathrm{I} 8 \mathrm{~B}$ & {$[3]$} \\
\hline VP35 & Unvalidated experimentally (in silico methods) & 4IJE & {$[3-5]$} \\
\hline VP40 & Unvalidated experimentally (in silico methods) & $1 \mathrm{H} 2 \mathrm{C}$ & {$[3-6]$} \\
\hline NP & Not reported & $4 \mathrm{Z9P}$ & {$[7]$. } \\
\hline RdRp & Not reported & Not available & {$[8]$} \\
\hline
\end{tabular}

${ }^{a}$ Targets mean biological targets. Structures were retrieved from PDB. Ref., references: [1] Joob \& Wiwanitkit 2014; [2] Manicassamy et al. 2005; [3] Setlur et al. 2016; [4] Shah et al. 2015; [5] Palamthodi et al. 2012; [6] Tamilvanan \& Hopper 2013; [7] Rahangdale et al. 2015; [8] Islam et al. 2016.

Wang et al. (2011) studied the receptor binding domain of the GP of the EboV and its role in viral entry. Previous studies have identified the N-terminal region of the GP1 as crucial for receptor binding. Results showed that Arg64 and Lys95 were involved in receptor binding (Wang et al. 2011). Putative receptor binding residues were also identified and reported in this study (Wang et al. 2011). Manicassamy et al. (2005) investigated the role of the EboV GP1 in viral entry. The putative binding site residues perceived to be involved in receptor binding were Asp55, Leu57, Leu63, Arg64, Phe88, Lys95 and Ile170 (Manicassamy et al. 2005). Results from this study also suggest the N-terminal 150 residues in mature GP1 as the receptor binding domain (RBD) (residues 33-185).

In their transduction study, Manicassamy et al. (2005) performed site-directed mutagenesis analysis on the EboV GP $\Delta \mathrm{O}$. They classified the region between residues 57 and 64 as well as residues Phe88, Lys95 and Ile170 as part of the RBD (Manicassamy et al. 2005). Brindley et al. (2007) investigated important residues required for the binding and post binding of the EboV GP1. Residues Gly87/Phe88, Lys114/Lys115, Lys140, Gly143 and Tyr162 were found to be important for receptor binding (Brindley et al. 2007).

Palamthodi et al. (2012) identified drug lead molecules against the EboV. The structural information of the target proteins VP40 and VP35 was obtained from Protein Data Bank (PDB) and their active sites were determined using PyMOL (Palamthodi et al. 2012). Major active site residues of VP40 were Leu304, Ile305, Thr306 and Gln307. Active residues of VP35 include Lys309, Arg312, Lys339, Lys319, Arg322 and Arg305 (Palamthodi et al. 2012).

In a study by Tamilvanan \& Hopper (2013), high throughput virtual screening and docking studies were carried out on VP40 of the EboV. Grid boxes were generated and positions and size of the active site were represented by receptor grids. Grid point's level for the x, y, $\mathrm{z}$ axes were $3.68,19.15$ and 25.87, respectively. Thr123, Phe125 and Arg134 residues were within the gridbox. Grid generation was performed using optimized potential for liquid simulations (Tamilvanan \& Hopper 2013). Wiwanitkit (2014) studied the binding site prediction within EboV VP40. There are detected 17 binding sites within the VP40 protein, however, the positions are not precisely defined in the study (Wiwanitkit 2014). These predicted sites can be further used as targets for antiviral drug development (Wiwanitkit 2014).

In a study by Shah et al. (2015), the active site of three Ebola viral receptors were carried out using the computed atlas of surface topography of proteins (CASTp) server and visualized in Discovery studio visualizer 4.0 (Shah et al. 2015). The active site residues obtained for VP40 (PDB code: 1H2D) were identified as Gln159, Arg148, Arg151, Leu168, Asn130 and Pro131. VP24 (PDB code: 4M0Q) active sites were found to be Met149 and Glu46, whereas VP35 (3FKE) active site residues were Gln329, Val314, Ser317, Lys334, Thr335 and Aap252. Setlur et al. (2016) predicted the active site residues of four EboV proteins VP24 (PDB code: 4M0Q), VP30 (2I8B), VP35 (4IJE) and VP40 (1H2C) using the CASTp server using default parameters of the web server.

In a study by Rahangdale et al. (2015), the crystal structure of the NP of the EboV (PDB code: 4U2X) was applied. The coordinates for the active sites of the $\mathrm{NP}$ (4U2X) were defined as $\mathrm{X}=-15.04 ; \mathrm{Y}=-46.99$; $\mathrm{Z}=-28.39$ (volume of cavity $=1140.192$ ) Rahangdale et al. 2015). However, the authors failed to provide details concerning active site residues. The predicted active site of the RdRp of the EboV was generated using the CASTp server as indicated in a study by Islam et al. (2016), however, active site residues were also not reported there.

\section{EboV active site identification: further valida- tion}

Protein function can be linked to various diseases. The way, in which a protein functions, can be defined by its interactions with other molecules. In silico methods for the identification and characterization of the active sites of biological targets have become of great interest to researchers (Fukunishi \& Nakamura 2011). This can be attributed to a number of newly resolved structures whose molecular interactions and biochemical functions have been poorly characterized (Laurie \& Jackson 2005; Fukunishi \& Nakamura 2011). Due to the rapidly increasing number of known protein structures available, focus has been placed on the use of analytical tools as means of identifying the active sites of target pro- 
Table 3. Active site residues as determined using bioinformatics tools on biological targets of the EboV. ${ }^{a}$

\begin{tabular}{|c|c|c|c|c|c|c|}
\hline $\begin{array}{l}\text { Biological } \\
\text { targets }\end{array}$ & PDB & $\begin{array}{l}\text { Active site residues } \\
\text { from literature }\end{array}$ & $\mathrm{PDB}$ & $\begin{array}{l}\text { Active site residues } \\
\text { (Metapocket) }\end{array}$ & $\begin{array}{l}\text { Active site residues } \\
\text { (POOL) }\end{array}$ & $\begin{array}{l}\text { Graphical } \\
\text { representation }\end{array}$ \\
\hline \multirow[t]{2}{*}{ GP } & \multirow[t]{2}{*}{ Not reported } & R64; K95 [5] & \multirow[t]{2}{*}{$5 \mathrm{~F} 18$} & \multirow{2}{*}{$\begin{array}{l}\text { T108; L110; F165; G166; } \\
\text { G167; V169; L162; G163; } \\
\text { T164; I109; C107; N114; } \\
\text { N104; C161; N106; L100; } \\
\text { N118; Q117; P160; E79; } \\
\text { Q75; I76; Y22; H26; F27; } \\
\text { L97; P99; Y115; L151; F116 }\end{array}$} & \multirow{2}{*}{$\begin{array}{l}\text { Y115; G163; E79; } \\
\text { D159; H140; Y137; } \\
\text { D153; N114; D124; } \\
\text { Q117; N118; D136; } \\
\text { H120; H125; L113; } \\
\text { P160; H69; F116; D73; } \\
\text { L162; H138; C161; } \\
\text { L110; S119; E19 }\end{array}$} & \\
\hline & & $\begin{array}{l}\text { D55; L57; L63; R64; } \\
\text { F88; K95; I170 [6] } \\
\text { G87/F88; } \\
\text { K114/K115; K140; } \\
\text { G143; Y162 }\end{array}$ & & & & \\
\hline \multirow[t]{2}{*}{ VP24 } & 4M0Q [1] & M149; E46 [1] & \multirow[t]{2}{*}{$4 \mathrm{M} 0 \mathrm{Q}$} & \multirow[b]{2}{*}{$\begin{array}{l}\text { S151; K39; E46; D48; S155; } \\
\text { F47; A229; T226; L152; } \\
\text { F230; R154; L158; I45; } \\
\text { K228; T231; G44; L158; } \\
\text { N162; F230; I45; T231; } \\
\text { A229; G44; Y41; R154; } \\
\text { N162; I161; I157; E46; } \\
\text { S155; K228; S151; F47; } \\
\text { T226; D48; K39; L152; } \\
\text { S225; T34; Q36; G37; } \\
\text { TRP38; V49; T50; S225; } \\
\text { K159 }\end{array}$} & \multirow{2}{*}{$\begin{array}{l}\mathrm{R} 154 ; \mathrm{R} 154 ; \mathrm{Y} 41 ; \\
\mathrm{Y} 41 ; \mathrm{E} 46 ; \mathrm{E} 46 ; \mathrm{D} 25 ; \\
\mathrm{G} 44 ; \mathrm{E} 18 ; \mathrm{G} 44 ; \mathrm{K} 15 ; \\
\mathrm{I} 45 ; \mathrm{I} 45 ; \mathrm{A} 43 ; \mathrm{E} 18 ; \\
\mathrm{K} 14 ; \mathrm{C} 27 ; \mathrm{S} 155 ; \mathrm{CS} 27 ; \\
\mathrm{A} 43 ; \mathrm{K} 19 ; \mathrm{S} 151 ; \mathrm{D} 48 ; \\
\text { V31; T231 }\end{array}$} & \\
\hline & $4 \mathrm{M} 0 \mathrm{Q}[2]$ & $\begin{array}{l}\text { S155; L158; R154; } \\
\text { F230; T226; F47; } \\
\text { E46; S225; F230; } \\
\text { E46 [2] }\end{array}$ & & & & \\
\hline VP30 & $2 \mathrm{I} 8 \mathrm{~B}$ & $\begin{array}{l}\text { L247; S187; C174; } \\
\text { L144; C251; S182; } \\
\text { V176; K180; S182; } \\
\text { F181; Q185; T178 [2] }\end{array}$ & $2 \mathrm{I} 8 \mathrm{~B}$ & $\begin{array}{l}\text { L173; C174; F242; L243; } \\
\text { A246; T178; A151; F181; } \\
\text { V176; L247; L147; L144; } \\
\text { I148; L189; K180; R179; } \\
\text { T146; L249; Q185; L144; } \\
\text { S182; K183; H215; C251; } \\
\text { K251; Q185; S184; K180; } \\
\text { I148; S182; R179; F181; } \\
\text { S184; P250; L147; E252; } \\
\text { K183; L249; L188; K215; } \\
\text { V176; L173; T178; A151; } \\
\text { L247; A246; C174; F242; } \\
\text { L243 }\end{array}$ & $\begin{array}{l}\text { C251; K180; C251; } \\
\text { K180; C174; E252; } \\
\text { L249; P250; Y211; } \\
\text { F181; L144; M177; } \\
\text { F181; R179; M77; } \\
\text { E252; L144; L249; } \\
\text { H193; A246; Q185; } \\
\text { C174; R179; T143; } \\
\text { Y211 }\end{array}$ & \\
\hline \multirow[t]{3}{*}{ VP35 } & $3 \mathrm{FKE}[1]$ & $\begin{array}{l}\text { D252; Q329; V314; } \\
\text { S317; K334; T335 [1] }\end{array}$ & \multirow[t]{3}{*}{ 4IJE } & \multirow[b]{3}{*}{$\begin{array}{l}\text { D289; A319; D321; A322; } \\
\text { E262; A265; S266; E269; } \\
\text { R283; D271; Q279; C275; } \\
\text { I278; E269; K282; D271; } \\
\text { Q279; D321; R283; S266; } \\
\text { C275; I340; A322; G323; } \\
\text { T281; K282; I320; K339; } \\
\text { D271; E269; I278; E262; } \\
\text { TRP324; P285; I258; Q288; } \\
\text { R283; Q279; I340; S266; } \\
\text { G323; A319; D252; N254; } \\
\text { V284; S317; P318; P315; } \\
\text { S253; P316; T281; I320; } \\
\text { D289; P313; I286; K339; } \\
\text { V314; S255; P285; A312; } \\
\text { S255; I286; N254; S253; } \\
\text { E262; C275; I258; V284; } \\
\text { P285; I258; TRP324; P315; } \\
\text { Q288; L256; D257; A290; } \\
\text { A290; P316; S317; P318; } \\
\text { D289; S255; Q288; K282; } \\
\text { I286; Q329; HIS296; V294; } \\
\text { D252; S253; N254; T281; } \\
\text { P313; V314; P293; P292; } \\
\text { A291; G333; L256; D257; } \\
\text { D321; L249; T335; L330; } \\
\text { Q331; K248; V314; V245; } \\
\text { P315; P316; T335; I280; } \\
\text { A265; V325; L242; F263; } \\
\text { A276; S272; G270; G270; } \\
\text { S272; I278 }\end{array}$} & \multirow{3}{*}{$\begin{array}{l}\text { K339; D271; I340; } \\
\text { D289; R283; E262; } \\
\text { D321; D271; E269; } \\
\text { E269; K282; C275; } \\
\text { K339; C275; E262; } \\
\text { I258; A322; Q288; } \\
\text { D257; V284; Q279; } \\
\text { R283; A319; TRP324; } \\
\text { S317 }\end{array}$} & \\
\hline & Not reported [3] & $\begin{array}{l}\text { K309; R312; K339; } \\
\text { K319; R322; R305 [3] }\end{array}$ & & & & \\
\hline & 4IJE [2] & $\begin{array}{l}\text { L267; G270; D321; } \\
\text { A322; S310; S255; } \\
\text { V245; T237; K339; } \\
\text { P316; I286; I258; } \\
\text { S255; K251 [2] }\end{array}$ & & & & \\
\hline
\end{tabular}


Table 3. (continued)

\begin{tabular}{|c|c|c|c|c|c|c|}
\hline $\begin{array}{l}\text { Biological } \\
\text { targets }\end{array}$ & $\mathrm{PDB}$ & $\begin{array}{l}\text { Active site residues } \\
\text { from literature }\end{array}$ & $\mathrm{PDB}$ & $\begin{array}{l}\text { Active site residues } \\
\text { (Metapocket) }\end{array}$ & $\begin{array}{l}\text { Active site residues } \\
\text { (POOL) }\end{array}$ & $\begin{array}{l}\text { Graphical } \\
\text { representation }\end{array}$ \\
\hline \multirow[t]{4}{*}{ VP40 } & \multicolumn{2}{|l|}{$1 \mathrm{H} 2 \mathrm{D}[1]$} & \multirow[t]{4}{*}{$1 \mathrm{H} 2 \mathrm{C}$} & \multirow{4}{*}{$\begin{array}{l}\text { H124; P31; Q159; E160; } \\
\text { L163; V166; L168; G12; } \\
\text { Q170; Y171; F125; K127; } \\
\text { A128; Q167; P169; N130; } \\
\text { F172; T129; L158; P165; } \\
\text { P164 }\end{array}$} & \multirow{4}{*}{$\begin{array}{l}\text { Y120; R137; L138; } \\
\text { S119; T121; H124; } \\
\text { N136; G139; A118; } \\
\text { R134; L117; K127; } \\
\text { R148; N154; H145; } \\
\text { P140; G153; M116; } \\
\text { I115; Q155; L149; } \\
\text { L176; D102; V135; } \\
\text { Y106 }\end{array}$} & \\
\hline & Not reported [3] & $\begin{array}{l}\text { Q159; R148; R151; } \\
\text { L168; N130; P131; } \\
\text { L304; I305; T306; } \\
\text { Q307 [3] }\end{array}$ & & & & \\
\hline & $1 \mathrm{H} 2 \mathrm{C}[2]$ & $\begin{array}{l}\text { P164; Q167; P93; } \\
\text { W95; Q170; A128; } \\
\text { P131; F125 [2] }\end{array}$ & & & & \\
\hline & Not reported $[4]$ & $208 ; 216 ; 249[4]$ & & & & \\
\hline NP & - & - & 4Z9P & $\begin{array}{l}\text { M198; V199; R202; L203; } \\
\text { G63; F60; A62; V64; T206; } \\
\text { I200; D65; F66; Q67; N207; } \\
\text { A70; F208; E68; D71; S69; } \\
\text { L209; R39; L300; N301; } \\
\text { L302; S303; F104; I41; } \\
\text { H290; Y98; F212; Y293; } \\
\text { H102; L99; V40; P42; S72; } \\
\text { P122; Y44; G103; R37; } \\
\text { K289; A35; Q38; G101; } \\
\text { V36; F106; L75 }\end{array}$ & $\begin{array}{l}\text { C78; H216; Y83; } \\
\text { D229; H81; H220; } \\
\text { Q217; K211; D71; } \\
\text { L213; C53; A82; L214; } \\
\text { Y293; F212; L209; } \\
\text { E292; D226; H80; } \\
\text { Y98; L300; I210; F208; } \\
\text { R298; H102 }\end{array}$ & \\
\hline
\end{tabular}

${ }^{a}$ PDB means Protein Data Bank code. References: [1], Shah et al. (2015); [2], Setlur et al. (2016); [3], Palamthodi et al. (2012); [4], Joob \& Wiwanitkit (2016); [5], Wang et al. (2011); [6], Manicassamy et al. (2005). Amino acids highlighted in green and yellow indicate an overlap of active site residues as per literature and online tools.

teins (Laurie \& Jackson 2005). The precise description of active sites of biological targets remains a challenge (Fukunishi \& Nakamura 2011).

The advent of bioinformatics tools have enabled the identification of such active sites with many approaches being developed and used in literature as well as our own reports (Chetty \& Soliman 2014; Arodola \& Soliman 2015). PDB present a number of 3D crystal structures of protein targets (Kamdar \& Dumontier 2015), which are increasing at a rapid rate, however, data on experimental protein-ligand binding for novel protein targets and their inhibitors are lacking. It is for this reason that active site residues need to be identified to enable docking of potential inhibitors to the active site (Laurie \& Jackson 2005). Identification, description and understanding of potential binding pockets on the 3D structure of the protein is a critical starting point and, if performed accurately, it can successfully pave the way for the development of novel drugs or inhibitors. If the correct binding site cannot be identified then any effort to validate it using techniques, such as molecular docking, molecular dynamics (MD) and other in silico approaches would be rendered futile. Hence, this report aims to further elaborate on studies in literature concerning the active sites of the biological targets of the EboV.

There currently exists a number of computational methods that can be utilized to identify active site residues. The crystal structure of EboV proteins in complex with bound inhibitors was reported and is available in PDB. Active site identification of EboV proteins has also been identified using unvalidated in silico methods as per literature. Most of the published reports use only one method of active site identification without any validation, hence, the outcome could be questionable. To further validate the outcome of the reported active site residues, we use "cross validation" as a means to provide more reliable and accurate results. In this study we opted to use two programs: (i) MetaPocket (Huang et al. 2010; Zhang et al. 2011); and (ii) Partial Order Optimum Likelihood (POOL) (Somarowthu \& Ondrechen 2012) - as the methods of choice to aid in the identification of possible binding sites on the EboV proteins. MetaPocket 2.0 (Heo et al. 2014), a consensus method that combines 8 different energy and geometry-based predictors, whereas the software POOL relates functional binding sites to sequence (Somarowthu \& Ondrechen 2012). It predicts catalytic residues using evolutionary, electrostatic as well as pocket geometric information. Geometry cleft sizes can be determined by this program using ConCavity (Capra et al. 2009) and sequence alignment-based conservation scores can be identified using an information-theoretic approach for functional site identification (INTREPID) (Sankararaman \& Sjölander 2008; Somarowthu et al. 2011). The crystal structures of the six EboV proteins were obtained from $\mathrm{PDB}$ as from the basic search tool. The results obtained from MetaPocket and POOL established the locality of possible binding pockets of the EboV proteins (Chetty \& Soliman 2014). Following the identification of each pocket, the pocket size can then be determined, which takes into account the surface area, volume and depth as well as surface atoms and potential interacting residues (Saberi Fathi \& Tuszyn- 
ski 2014). The data represented below highlights the best active sites and active site residue numbers of all the proteins of the EboV.

Table 3 highlights the active site residues obtained in our study using two different bioinformatics tools. On comparison of the results generated using MetaPocket and POOL, it can be seen that the active site residues do overlap based on their similarity, however, it was also observed that a number of these active site residues do not share any similarity, as determined using the two softwares. It was also interesting to note that active site residues, as reported in literature, were similar to that identified in our study with the exception of a few. The identification of active site residues is a crucial and fundamental step towards the design of EboV inhibitors. Subsequent to the EboV protein homology model and target site determination, several paths may be utilized in the development of inhibitors. The challenge that the identification of active sites residues present has prompted us to further investigate and enrich the current information concerning this. This study will offer a step forward towards providing valuable information that will fill in the gap of knowledge in this area.

A huge emphasis is currently placed on the investigation of host proteins and druggable viral targets (Omotuyi 2015). An in-depth analysis of the biological targets of the EboV is required for binding site identification (Joob \& Wiwanitkit 2014). This can be achieved by a complex crystallography study. With the increasing efforts towards understanding and applying bioinformatics technology, it is possible for further manipulation of the crystal structure of EboV proteins. This in turn will allow further analysis of the druggability within the identified active pocket (Joob \& Wiwanitkit 2014). In order to expand the search for new drugs, a sound knowledge of viral protein structures is essential. The identification of active sites of biological targets is a critical process prompting further studies towards the design and discovery of anti-Ebola drugs.

\section{Homology modeling}

In the absence of an experimentally determined crystal structure, homology modeling provides a useful 3D model for a protein of interest that is related to that of a known protein structure (Eswar et al. 2007). Protein modeling plays a pivotal role in the process of drug discovery. The goal being to predict a structure from its sequence with an accuracy that is comparable to the best results achieved experimentally (Bishop et al. 2008; Samant et al. 2014). However, there is a number of restrictions associated with homology modeling and these include limitations in the availability of $3 \mathrm{D}$ crystal structures, as well as the presence of loops and inserts (Bishop et al. 2008; Honarparvar et al. 2014). The quality and accuracy of a homology model is also dependent on a number of different factors and these include a high resolution experimental protein structure required as a template, the degree of sequence identity between the query and template sequence and alignment errors in- crease rapidly when sequence identity is less than $30 \%$. A medium accurate model requires a sequence identity of between 30-50\% (Bishop et al. 2008).

With a vast array of DNA sequencing techniques available providing researchers with a wealth of knowledge concerning genomic data, experimental structure determination techniques require further efforts. Subsequently the gap between the number of known protein sequences and protein structures are growing at an increasing rate. Thus, in order to fill in the gap, a number of computational approaches have been developed to predict the tertiary structure of a protein using its amino acid sequence (Mariani et al. 2013; Prashat 2015). In our lab we use various methods to generate 3D crystal structures of unresolved proteins (Fig. 1), and this can be seen in our publications (Maharaj \& Soliman 2013; Chetty \& Soliman 2014; Moonsamy et al. 2014; Arodola \& Soliman 2015). One of the most prominent and promising approaches is comparative modeling of a protein (homology modeling), a most reliable method used to predict and construct a plausible 3D structure of a target protein.

Despite many targets being resolved, there still exits a few of which no crystal structure is available (Table 1). This has prompted us to attempt to build a homology-based 3D model for our protein of interest. This model has been put forth as a guideline to assist researchers to conduct future studies. BLASTp was performed against PDB to retrieve the best suitable templates for modeling the protein of interest. The template of choice contains the maximum sequence identity and lowest e-value. The modeling of the 3D structure of the protein was performed using an online software tool, RaptorX. RaptorX, a protein structure prediction server established by the Xu's group was found to be valuable in predicting the $3 \mathrm{D}$ structure of a protein sequence devoid of protein homologs from the PDB (Källberg et al. 2012). Following the submission of an input query sequence, the software predicts both the secondary and tertiary structures together with solvent accessibility and disordered regions. It also assigns confidence scores to indicate the quality of the predicted model (Källberg et al. 2012).

Once the 3D structure of the protein of interest has been modeled and target sites identified, a number of different strategies can then be put into place towards developing potential inhibitors. If the drug target is an enzyme, a designed chemical molecule of choice may be able to fit within the active site pocket. In view of these homology modeling methods offered, the only possible way at present is to obtain structural information for a large number of proteins. In silico methods could prove to be useful in assisting researchers as well as medicinal chemists towards the design of potential inhibitors by analyzing the physiochemical and structural properties of a protein (Prashat 2015).

\section{Homology model of RdRp of the EboV}

In order for computer-aided drug design (CADD) of 


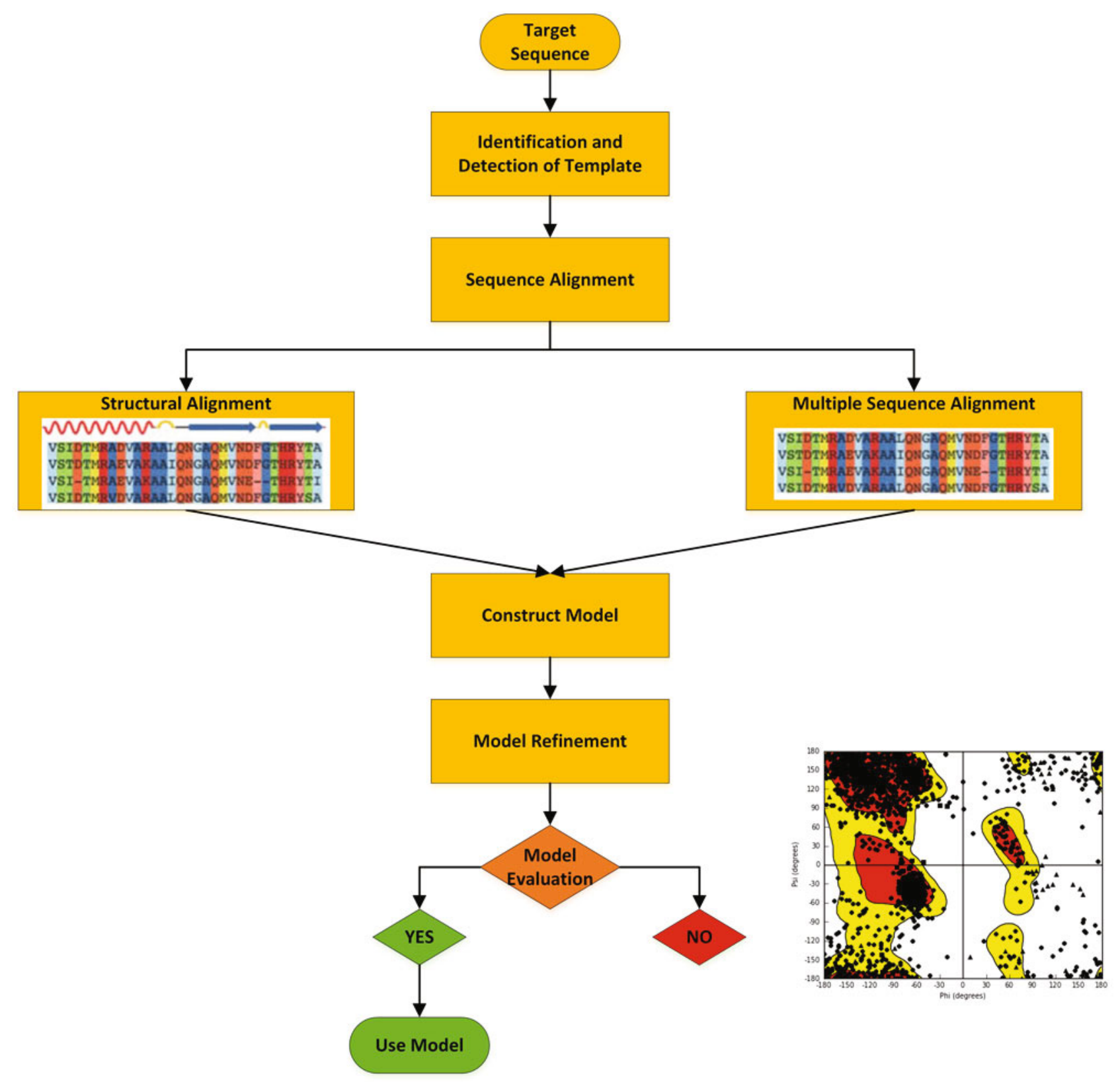

Fig. 1. Highlights of the homology modeling protocol we adopted in our research group (Maharaj \& Soliman 2013; Chetty \& Soliman 2014; Moonsamy et al. 2014; Arodola \& Soliman 2015).

Ebola inhibitors to occur, a 3D crystal structure of a target protein is needed. Reduced availability of experimentally determined target protein structure, $\mathrm{RdRp}$ proved to be a limitation in this study. Theoretical models built by homology modeling have served as a practical and effective alternative of this unresolved protein. The tertiary structure of RdRp was not available in $\mathrm{PDB}$, therefore the complete EboV protein sequence was retrieved from the National Centre for Biotechnology Information (accession No.: NP_066251.1) and submitted to RaptorX for the generation of a 3D homology model (Fig. 2). The quality and validation of the generated model was done using a Ramachandran plot analysis using Maestro (http://www.schrodinger.com/) (Schrödinger 2013). Results from the Ramachandran plot revealed that $84.1 \%(1859 / 2210)$ of all residues were in favored (98\%) regions and 94.0\% (2078/2210) of all residues were in allowed $(>99.8 \%)$ regions.

Results from our study reveal that RaptorX provided a reliable and favorable result on the basis of structure prediction and validation using the Ramachandran plot (Fig. 2A). The homology model generated using RaptorX was therefore selected as a model of choice and utilized for further computational analysis. The homology model was superimposed with the template 5A22_A using Swiss-Pdb Viewer (v4.1.0). As can be seen in Figure 2B, the structure presents a few outliers which mainly lie outside the active site region of the protein. However, despite the low percentage of sequence identity with the template $(22 \%)$ this homology model can be accepted based on the superimposition, as it aligns well with the template protein 5A22_A. 
A

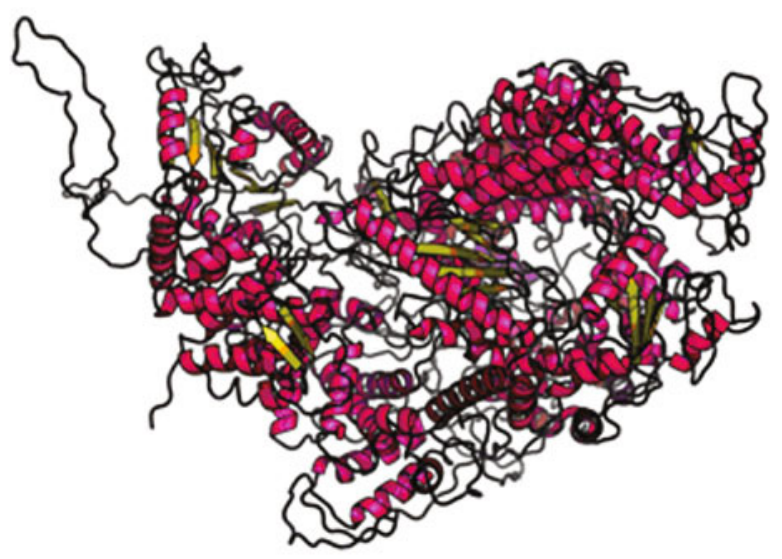

B

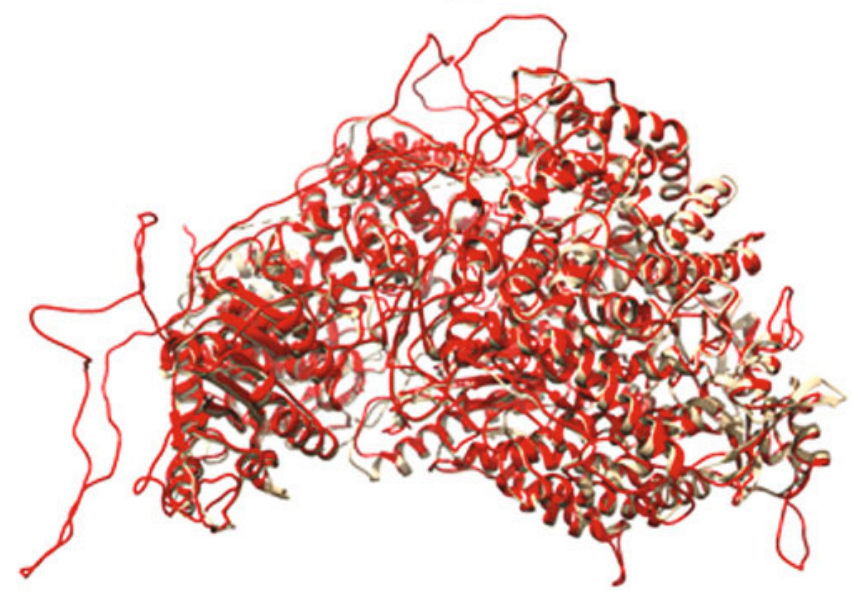

Fig. 2. (A) Homology model of the RdRp generated using RaptorX. (B) Superimposition of the homology model (red) generated using RaptorX with template protein (grey; PDB code: 5A22) using the Swiss-Pdb Viewer.

\section{Docking-guided active site determination}

RaptorX is used for secondary protein structure prediction, template-based tertiary structure modeling, alignment quality assessment as well as sophisticated probabilistic alignment sampling (Källberg et al. 2012). This software distinguishes itself from other servers and hence chosen based on the accuracy of alignment between a target sequence, one or multiple distantly related template proteins, by a novel nonlinear scoring function and a probabilistic-consistency algorithm (Källberg et al. 2012). Subsequently, RaptorX provides high-quality structural models for a number of unresolved targets (Källberg et al. 2012). The RdRp sequence has been analyzed and a homology model generated using RaptorX. However, due to this protein consisting of more than 2,000 amino acid residues, active site determination proved to be difficult and this has also been reported in a recent publication (Grifoni et al. 2016). Therefore, we embarked on a different approach to identify possible active site residues using dockingguided active site determination. Molecular docking is a method that is used to predict the binding affinity as well as the position of the ligand in the active site pocket (Kumalo et al. 2015; Ramesh et al. 2016). Here we employ docking in order to identify the location of possible active sites as well as protein-ligand binding interactions, which play a critical role in the development of novel EboV inhibitors. This method provides an account of the calculated binding free energies at the different docking sites. Docking studies were carried out as per our previous publications (Chetty \& Soliman 2014; Cele et al. 2016) using the homology model of RdRp with an experimentally reported inhibitor, Favipiravir (T-705). However, it is unclear exactly where on the protein the inhibitor binds. Favipiravir (T-705), a pyrazine derivative, was found to be potent against the influenza virus. It is active against a range of influenza viruses as well as other RNA viruses (Harris \& Pierpoint 2012). Recently, it has been shown to inhibit EboV infection in cell culture, as well as reduce viremia and cell death, making it a promising target for the treatment of Ebola (Harris \& Pierpoint 2012). Grooves of the protein were identified and the grid box was defined as large as possible to allow for more runs and increase the search space. Binding energies were then compared and the best conformation was selected. This approach is more reasonably valid based on our previous methods as per our previous publications (Chetty \& Soliman 2014; Kumalo \& Soliman 2015; Ramesh et al. 2016).

\section{Docking calculations}

Docking studies were performed on the retrieved ligand Favipiravir (T-705) (zinc code 13915654), in a mol2 format, downloaded from the zinc database (https://docking.org/; Irwin \& Shoichet 2005). The ligand was assessed in Molegro Molecular Viewer (http://www.molegro.com/) to ensure that it displayed the correct bond angles and hybridization state. AutoDock Tools (Morris \& Huey 2009) graphical user interface was used to define the gridbox at different positions on the protein as the active site was unknown and not documented in literature. The gridbox measured in Angstroms for sites 1-3 was defined using the following parameters: site 1 , centre $(\mathrm{X}=11.362, \mathrm{Y}=$ $-0.828, \mathrm{Z}=33.439)$ and dimensions $(\mathrm{X}=126, \mathrm{Y}=126$, $\mathrm{Z}=126)$; site 2 , centre $(\mathrm{X}=15.182, \mathrm{Y}=-9.253, \mathrm{Z}=$ 40.559) and dimensions $(\mathrm{X}=126, \mathrm{Y}=126, \mathrm{Z}=126)$; site 3 , centre $(\mathrm{X}=-15.767, \mathrm{Y}=9.186, \mathrm{Z}=34.694)$ and dimensions $(\mathrm{X}=126, \mathrm{Y}=126, \mathrm{Z}=126)$. The exhaustiveness of the system was set at 500 and the number of modes at 20. Docking calculations using the Lamarkian genetic algorithm were performed with AutoDock Vina (Morris \& Huey 2009).

Docking results revealed that the ligand Favipiravir (T-705) bound to the same area of the protein with closely related binding energies (Table 4). Visual inspection of the docked results, together with the dock- 


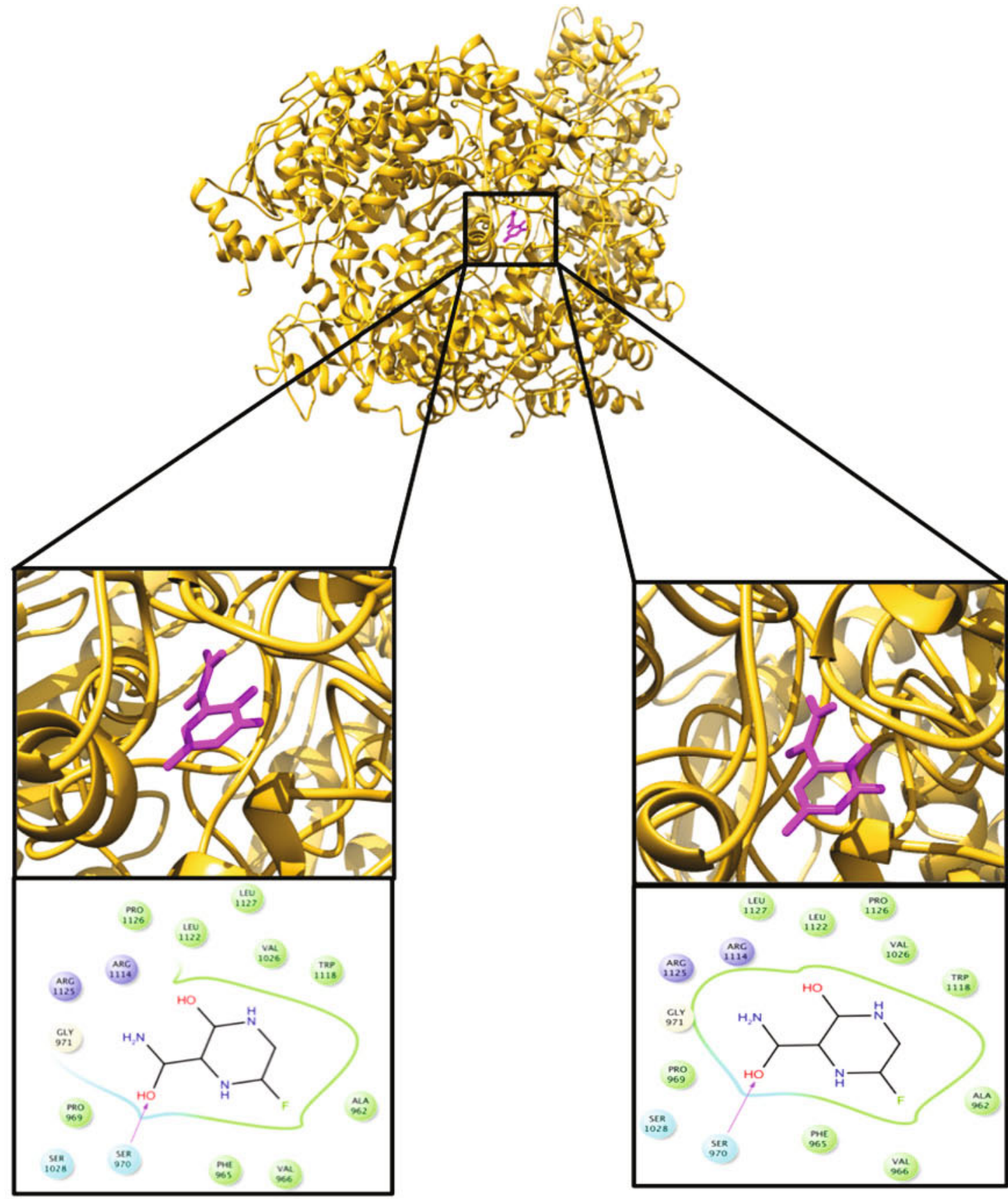

Sites 1 and 2

Site 3

$\Delta \mathrm{G}=-7.2 \mathrm{kcal} / \mathrm{mol}$

$\Delta \mathrm{G}=-7.1 \mathrm{kcal} / \mathrm{mol}$

\begin{tabular}{|c|c|c|c|c|}
\hline Charged (negative & $\mathrm{H}_{2} \mathrm{O}$ & Water & $\rightarrow$ & $\mathrm{H}$-bond (side chain) \\
\hline Charged (positive) & 0 & Hydration site & $\longrightarrow$ & Metal coordination \\
\hline Polar & $\infty$ & Displaced hydration site & - & Salt Bridge \\
\hline Hydrophobic & $\bullet$ & $\pi-\pi$ stacking & & Solvent exposure \\
\hline Glycine & $\rightarrow$ & $\pi$-cation & & \\
\hline Metal & $\rightarrow$ & $\mathrm{H}$-bond (backbone) & & \\
\hline
\end{tabular}

Fig. 3. Docked complexes and two-dimensional interaction maps at sites S1-S3 on the protein.

ing scores indicated that the ligand fitted better at site 1 and site 2 as can be seen in Table 4 and Figure 3.

The difference in the docking results for sites
1 and $2(-7.2 \mathrm{kcal} / \mathrm{mol})$ in comparison to site 3 $(-7.1 \mathrm{kcal} / \mathrm{mol})$ is almost negligible. Based on docking results and relative binding energy obtained for the 
Table 4. Docking results showing binding free energies of the Favipiravir (T-705) at the three different sites (S1-S3).

\begin{tabular}{llcc}
\hline \multirow{2}{*}{ Inhibitor } & \multicolumn{3}{c}{ Docking scores $(\mathrm{kcal} / \mathrm{mol})$} \\
\cline { 2 - 4 } & $\mathrm{S} 1$ & $\mathrm{~S} 2$ & $\mathrm{~S} 3$ \\
\hline Favipiravir (T-705) & -7.2 & -7.2 & -7.1 \\
\hline
\end{tabular}

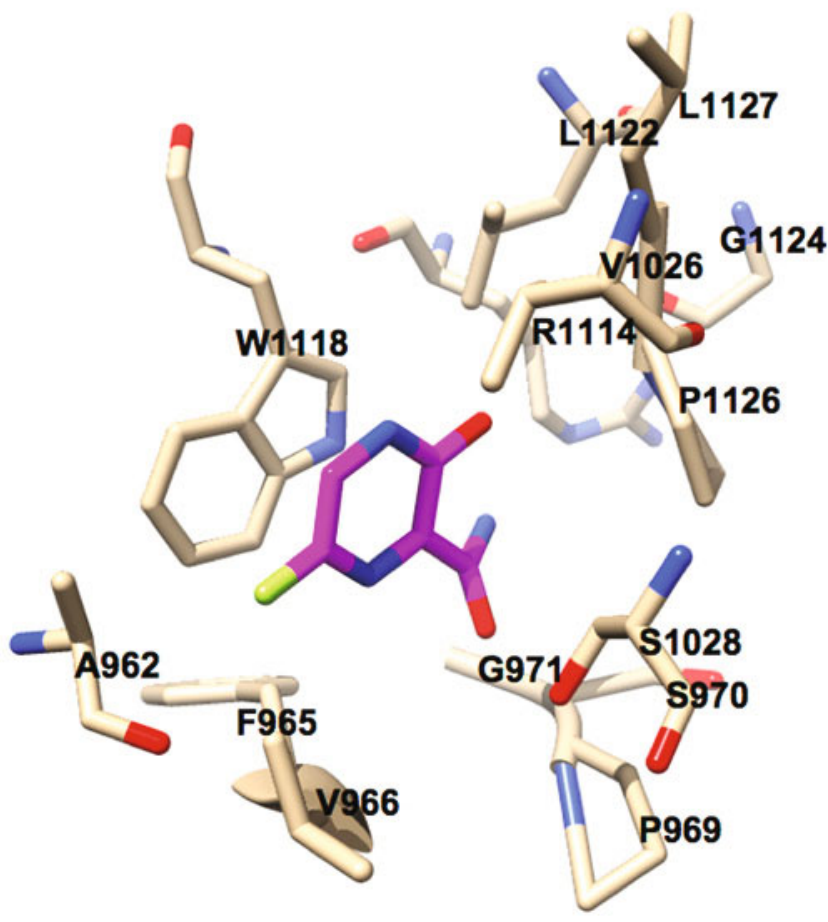

Fig. 4. Interacting residues of RdRp with Favipiravir (T-705) at binding site 1 .

three sites, site 1 and site 2 can be selected as the possible active sites on the protein for the binding of Favipiravir (T-705) as it resulted in the highest binding energy $(-7.2 \mathrm{kcal} / \mathrm{mol})$.

Ligand interaction plots (Fig. 3) provided us with the predicted interactions of the ligand at each selected docking site. Figures 3 and 4 provide a summary of the interaction of the ligand with residues of the $\mathrm{RdRp}$ at potential binding sites (S1-S3). A hydrogen bond occurred between the polar residue Ser970 and the hydroxyl group of the ligand for sites 1,2 and 3. Polar amino acids, Ser970 and Ser1028 contributed to the polar solvation energy. Gly971 contributes to the electrostatic and polar solvation energies. Residues Pro1126, Leu1122, Leu1127, Val1026, Trp1118, Ala962, Val966, Phe965 and Pro969 participate in hydrophobic interactions, thereby contributing to the overall van der Waals energy. Arg1114 and Arg1125 are positively charged amino acids that contribute to the electrostatic and polar solvation energies. In general, a large number of hydrophobic interactions were observed from the binding of the protein and ligand following docking.
Despite docking being efficient, the limitation surrounding this method stems from the lack of a universally adopted standard for scoring functions Warren et al. 2006). Another factor, which contributes to the reliability and accuracy of results has a lot to do with the confirmation of the receptor that has been selected (Kitchen et al. 2004; Alonso et al. 2006), thus docking alone cannot provide reliable results (Arodola \& Soliman 2015). Further validation methods, such as MD simulations can be used to aid in the understanding of the dynamic nature of a biomolecule at different time frames (Hansson et al. 2002; Alonso et al. 2006), as well as to obtain the binding free energies, which are more accurate than those obtained from docking alone (Alonso et al. 2006). These results provide critical information concerning the nature of the binding site and the key protein-ligand interactions that are responsible for the molecular recognition serving as a validation tool.

In-silico route map towards the design and discovery of EboV inhibitors

Prompted by limited experimental and in silico research into potential EboV inhibitors, we put forth a beneficial route map (Fig. 5), which will highlight the various techniques that may be successfully utilized towards EboV drug design. CADD is a representation of computational methods that are implemented towards the design and discovery of new therapeutic solutions (Song et al. 2009). There exists a number of bioinformatics tools, which have aided in the advancement of the drug discovery process (Bamborough \& Cohen 1996; Anderson 2003). The recent improvements made in computational chemistry software, CADD and MD simulations, have led to innovative research methods in the pharmaceutical industry (Huang et al. 2010).

\section{Conclusion}

The current EboV outbreak has highlighted the need for effective anti-filoviral therapeutic agents. The identification of ligand-receptor binding sites is crucial for any structure-based drug design. Apart from elucidating the functional characterization of a protein, a sound knowledge of the potential binding sites can guide researchers towards the design of inhibitors. We suggest using multiple software for the identification of potential active site residues on EboV targets, as the ones reported in our study are not entirely consistent to what has been previously documented. These can then be validated using computational methods, such as molecular docking, MD simulations as well as thermodynamics like free binding energy and entropy calculations. Easily accessible tools and servers are available, which can be utilized to gain further insight on proteins in three-dimensions as well as to gather in-depth information about proteins using in silico approaches. For example, molecular docking, which yielded a top score of $-7.2 \mathrm{kcal} / \mathrm{mol}$ at sites 1 and 2 suggesting that these 


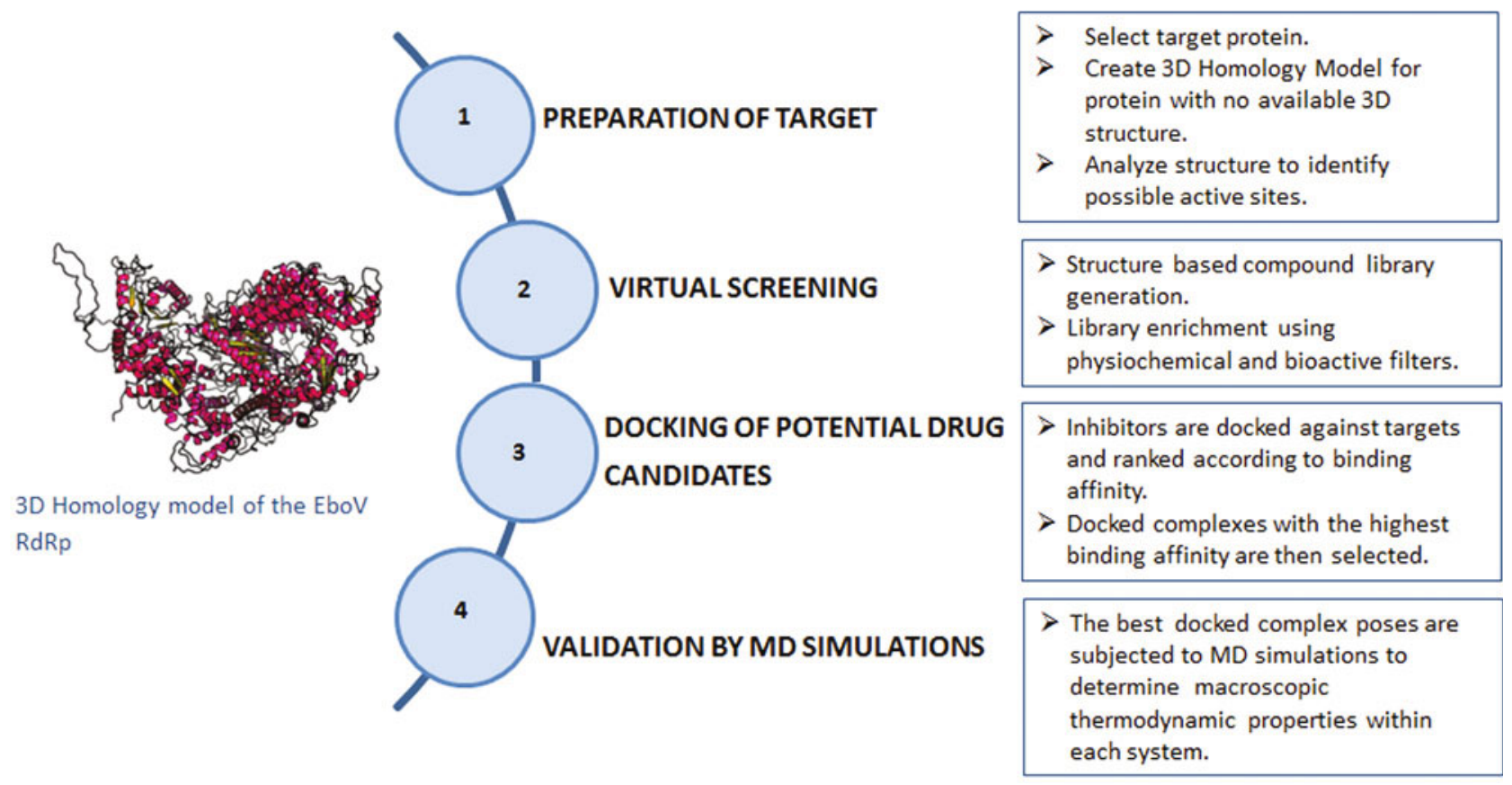

Fig. 5. Route map toward the in-silico design of EboV inhibitors using the homology modeled RdRp protein.

could be the possible active sites on the protein for the binding of Favipiravir (T-705), a promising inhibitor. It is also interesting to note that Favipiravir (T-705) forms a strong hydrogen bond with Ser970 as can be seen from the two-dimensional interaction map. This research provides a valuable platform in assisting researchers to provide a better understanding towards further experimental and computational efforts to design novel drugs and inhibitors that could effectively target disease-related proteins and eradicate this deadly virus.

\section{Acknowledgements}

The authors acknowledge the College of Health Sciences, UKZN, and the National Research Foundation for their financial support.

\section{Conflicts of interest}

Authors declare that there are no conflicts of interest.

\section{References}

Alonso H., Bliznyuk A.A. \& Gready J.E. 2006. Combining docking and molecular dynamic simulations in drug design. Med. Res. Rev. 26: 531-568.

Anderson A.C. 2003. The process of structure-based drug design. Chem. Biol. 10: 787-797.

Arodola O.A. \& Soliman M.E.S. 2015. Could the FDA-approved anti-HIV PR inhibitors be promising anticancer agents? An answer from enhanced docking approach and molecular dynamics analyses. Drug Des. Devel. Ther. 9: 6055-6065.

Bamborough P. \& Cohen F.E. 1996. Modeling protein-ligand complexes. Curr. Opin. Struct. Biol. 6: 236-241.

Bishop A.O.T., de Beer T.A.P. \& Joubert F. 2008. Protein homology modelling and its use in South Africa. South Afr. J. Sci. 104: 2-6.

Brindley M., Hughes L., Ruiz A., McCray P. B., Sanchez A., Sanders D. A. \& Maury W. 2007. Ebola virus glycoprotein
1: identification of residues important for binding and postbinding events. J. Virol. 81: 7702-9.

Capra J.A., Laskowski R.A., Thornton J.M., Singh M. \& Funkhouser T.A. 2009. Predicting protein ligand binding sites by combining evolutionary sequence conservation and 3D structure. PLoS Comput. Biol. 5: e1000585.

Cele F.N, Muthusamy R. \& Soliman M.E. 2016. Per-residue energy decomposition pharmacophore model to enhance virtual screening in drug discovery?: a study for identification of reverse transcriptase inhibitors as potential anti-HIV agents. Drug Des. Devel. Ther. 10: 1365-1377.

Chetty S. \& Soliman M.E.S. 2014. Possible allosteric binding site on Gyrase B, a key target for novel anti-TB drugs: homology modelling and binding site identification using molecular dynamics simulation and binding free energy calculations. Med. Chem. Res. 24: 2055-2074.

Chiappelli F., Bakhordarian A., Thames A.D., Du A.M., Jan A.L., Nahcivan M., Nguyen M.T., Sama N., Manfrini E., Piva F., Rocha R. \& Maida C.A. 2015. Ebola: translational science considerations. J. Transl. Med. 13: 11.

Dahms S.O., Hardes K., Becker G.L., Steinmetzer T., Brandstetter H. \& Than M.E. 2014. X-ray structures of human furin in complex with competitive inhibitors. ACS Chem. Biol. 9: $1113-1118$.

Eswar N., Webb B., Marti-Renom M.A., Madhusudhan M.S., Eramian D., Shen M.Y., Pieper U. \& Sali A. 2007. Comparative protein structure modeling using MODELLER. Curr. Protoc. Protein Sci. Chapter 2, Unit 2.9.

Feldmann H. \& Geisbert T.W. 2011. Ebola haemorrhagic fever. Lancet 377: 849-862.

Fukunishi Y. \& Nakamura H. 2011. Prediction of ligand-binding sites of proteins by molecular docking calculation for a random ligand library. Protein Sci. 20: 95-106.

Gebre Y., Gebre T. \& Peters A. 2014. The Ebola virus: a review of progress and development in research. Asian Pac. J. Trop. Biomed. 4: 928-936.

Grifoni A., Lo Presti A., Giovanetti M., Montesano C., Amicosante M., Colizzi V., Lai A., Zehender G., Cella E., Angeletti S. \& Ciccozzi M. 2016. Genetic diversity in Ebola virus: phylogenetic and in silico structural studies of Ebola viral proteins. Asian Pac. J. Trop. Med. 9: 337-343.

Haasnoot J., De Vries W., Geutjes E.J., Prins M., De Haan P. \& Berkhout B. 2007. The Ebola virus VP35 protein is a suppressor of RNA silencing. PLoS Pathog. 3: e86. 
Hansson T., Oostenbrink C. \& van Gunsteren W.F. 2002. Molecular dynamics simulations. Curr. Opin. Struct. Biol. 12: 190.

Haque A., Hober D. \& Blondiaux J. 2015. Addressing therapeutic options for Ebola virus infection in current and future outbreaks. Antimicrob. Agents Chemother. 59: 5892-5902.

Harris F. \& Pierpoint L. 2012. Photodynamic therapy based on 5-aminolevulinic acid and its use as an antimicrobial agent. Med. Res. Rev. 29: 1292-1327.

Hartlieb B. \& Weissenhorn W. 2006. Filovirus assembly and budding. Virology 34: 64-70.

Heo L., Shin W.H., Lee M.S. \& Seok C. 2014. GalaxySite: ligandbinding-site prediction by using molecular docking. Nucleic Acids Res. 42: W210-W214.

Honarparvar B., Govender T., Maguire G.E.M., Soliman M.E.S. \& Kruger H.G. 2014. Integrated approach to structure-based enzymatic drug design: molecular modeling, spectroscopy, and experimental bioactivity. Chem. Rev. 114: 493-537.

Huang H.J., Yu H.W., Chen C.Y., Hsu C.H., Chen H.Y., Lee K.J., Tsai F.J. \& Chen C.Y.C. 2010. Current developments of computer-aided drug design. J. Taiwan Inst. Chem. Eng. 41: 623-635.

Huang Y., Xu L., Sun Y. \& Nabel G.J. 2002. The assembly of Ebola virus nucleocapsid requires virion-associated proteins 35 and 24 and posttranslational modification of nucleoprotein. Mol. Cell 10: 307-316.

Irwin J.J. \& Shoichet B.K. 2005. ZINC - a free database of commercially available compounds for virtual screening. J. Chem. Inf. Model. 45: 177-182.

Islam M. J., Fatema K. \& Biswas P. 2016. In silico approach of structure prediction and functional characterization of Zaire Ebola (EboV) and identification of binding site for drug development. Int. J. Appl. Sci. Biotechnol. 4: 92.

Johansen L.M., Brannan J.M., Delos S.E., Shoemaker C.J., Stossel A., Lear C., Hoffstrom B.G., Dewald L.E., Schornberg K.L., Scully C., Lehár, J., Hensley L.E., White J.M. \& Olinger G.G. 2013. FDA-approved selective estrogen receptor modulators inhibit Ebola virus infection. Sci. Transl. Med. 5: $190 \mathrm{ra79}$.

Joob B. \& Wiwanitkit V. 2014. Identification of active pocket and protein druggability within envelope glycoprotein GP2 from Ebola virus. Asian Pac. J. Trop. Biomed. 4: 939-940.

Joob B. \& Wiwanitkit V. 2016. Determination of ligand cluster and binding site within VP40 of Ebola virus: clue for drug development. Asian Pac. J. Trop. Med. 9: 410-411.

Kamdar M.R. \& Dumontier M. 2015. An Ebola virus-centered knowledge base. Database 2015: bav049.

Källberg M., Wang H, Wang S., Peng J., Wang Z., Lu H. \& Xu J. 2012.Template-based protein structure modeling using the RaptorX web server. Nat. Protocols 7: 1511-1522.

Kitchen D.B., Decornez H., Furr J.R. \& Bajorath J. 2004. Docking and scoring in virtual screening for drug discovery: methods and applications. Nat. Rev. Drug. Discov. 3: 935-949.

Kumalo H.M., Bhakat S. \& Soliman M.E. 2015. Heat-shock protein 90 (Hsp90) as anticancer target for drug discovery: an ample computational perspective. Chem. Biol. Drug Des. 86: 1131-1160.

Kumalo H.M. \& Soliman M.E. 2015. Per-residue energy footprints-based pharmacophore modeling as an enhanced in silico approach in drug discovery: a case study on the identification of novel $\beta$-secretase1 (bace1) inhibitors as anti-Alzheimer agents. Cell. Mol. Bioeng. 9: 175-189.

Lai K.Y., Ng W.Y.G. \& Cheng F.F. 2014. Human Ebola virus infection in West Africa: a review of available therapeutic agents that target different steps of the life cycle of Ebola virus. Infect. Dis. Poverty 3: 43.

Laurie A.T.R. \& Jackson R.M. 2005. Q-SiteFinder: an energybased method for the prediction of protein-ligand binding sites. Bioinformatics 21: 1908-1916.

Lee J.E., Fusco M.L., Hessell A.J., Oswald W.B. \& Burton D.R. 2009. Survivor antibody. Nature 454: 177-182.

Leung L.W., Park M.S., Martinez O., Valmas C., López C.B. \& Basler C.F. 2011. Ebolavirus VP35 suppresses IFN production from conventional but not plasmacytoid dendritic cells. Immunol. Cell Biol. 89: 792-802.
Maharaj Y. \& Soliman M.E. S. 2013. Identification of novel gyrase b inhibitors as potential anti-TB drugs: homology modelling, hybrid virtual screening and molecular dynamics simulations. Chem. Biol. Drug Des. 82: 205-215.

Manicassamy B., Wang J., Jiang H. \& Rong L. 2005. Comprehensive analysis of Ebola virus GP1 in viral entry comprehensive analysis of Ebola virus GP1 in viral entry. J. Virol. 79: 47934805.

Mariani V., Biasini M., Barbato A. \& Schwede T. 2013. IDDT: a local superposition-free score for comparing protein structures and models using distance difference tests. Bioinformatics 29: 2722-2728.

Martínez M.J., Salim A.M., Hurtado J.C. \& Kilgore P.E. 2015. Ebola virus infection: overview and update on prevention and treatment. Infect. Dis. Ther. 4: 365-390.

Moonsamy S., Dash R.C. \& Soliman M.E.S. 2014. Integrated computational tools for identification of CCR5 antagonists as potential HIV-1 entry inhibitors: homology modeling, virtual screening, molecular dynamics simulations and 3D QSAR analysis. Molecules 19: 5243-5265.

Morris G. \& Huey R., Lindstrom W., Sanner M.F., Belew R.K., Goodsell D.S. \& Olsen A.J. 2009. AutoDock4 and AutoDockTools4: automated docking with selective receptor flexibility. J. Comp. Chem. 30: 2785-2791.

Mühlberger E. 2013. NIH Public Access 2: 205-215.

Muyembe-Tamfum J.J., Mulangu S., Masumu J., Kayembe J.M., Kemp A. \& Paweska J.T. 2012. Ebola virus outbreaks in Africa: past and present. Onderstepoort J. Vet. Res. 79: 1-8.

Noda T., Halfmann P., Sagara H. \& Kawaoka Y. 2007. Regions in Ebola virus VP24 that are important for nucleocapsid formation. J. Infect. Dis. 196: S247-S250.

Noda T., Kolesnikova L. Becker S. \& Kawaoka Y. 2011. The importance of the NP: VP35 ratio in Ebola virus nucleocapsid formation. J. Infect. Dis. 204: 1-6.

Nyakatura E.K., Frei J.C. \& Lai J.R. 2015. Chemical and structural aspects of Ebola virus entry inhibitors. ACS Infect. 1: 42-52.

Okumura A., Pitha P.M., Yoshimura A. \& Harty R.N. 2010. Interaction between Ebola virus glycoprotein and host toll-like receptor 4 leads to induction of proinflammatory cytokines and SOCS1. J. Virol. 84: 27-33.

Omotuyi I.O. 2015. Ebola virus envelope glycoprotein derived peptide in human furin-bound state: computational studies. J. Biomol. Struct. Dyn. 33: 461-70.

Palamthodi S., Patil D., Sankpal A., Zarekar S. \& Patil Y. 2012. Identification of drug lead molecules against Ebola virus: an in silico approach. J. Comput. Methods Mol. Des. 2: 76-84.

Picazo E. \& Giordanetto F. 2014. Small molecule inhibitors of ebola virus infection. Drug Discov. Today 20: 277-286.

Rahangdale S., Malviya N.K., Singh Baghel A., Sharma P. \& Kale A. 2015. Screening of promising lead molecules against two drug targets in Ebola virus: an effort to eradicate Ebola infection. IOSR J. Pharm. Biol. Sci. Ver. III 10: 2319-7676.

Ramesh M., Vepuri S.B., Oosthuizen F. \& Soliman M.E. 2016. Adenosine monophosphate-activated protein kinase (AMPK) as a diverse therapeutic target: a computational perspective. Appl. Biochem. Biotechnol. 178: 810-830.

Rougeron V., Feldmann H., Grard G., Becker S. \& Leroy E.M. 2015. Ebola and Marburg haemorrhagic fever. J. Clin. Virol. 64: $111-119$.

Saberi Fathi S.M. \& Tuszynski J.A. 2014. A simple method for finding a protein's ligand-binding pockets. BMC Struct. Biol. 14: 18 .

Samant L.R., Sangar V.C. \& Chowdhary A. 2014. Online servers and offline tools for protein modelling, optimization and validation: a review. Int. J. Pharm. Sci. Rev. Res. 28: 123-127.

Sankararaman S. \& Sjölander K. 2008. INTREPID - INformation-theoretic TREe traversal for Protein functional site IDentification. Bioinformatics 24: 2445-52.

Schrödinger 2013. Innovations in computational drug design. Available at: http://www.schrodinger.com/ (accessed 28.09. 16).

Setlur A.S., Naik S.Y. \& Skariyachan S. 2016. Herbal lead as ideal bioactive compounds against probable drug targets of Ebola virus in comparison with known chemical analogue: 
a computational drug discovery perspective. Interdiscip. Sci. Comput. Life Sci. (in press) doi: 10.1007/s12539-016-0149-8.

Shah R., Panda P.K., Patel P. \& Panchal H. 2015. Pharmacophore based virtual screening and molecular docking studies of inherited compounds against Ebola virus receptor proteins. World J. Pharmacy Pharm. Sci. 4: 1268-1282.

Somarowthu S., Yang H., Hildebrand D.G.C. \& Ondrechen M.J. 2011. High-performance prediction of functional residues in proteins with machine learning and computed input features. Biopolymers 95: 390-400.

Somarowthu S. \& Ondrechen M.J. 2012. POOL server: machine learning application for functional site prediction in proteins. Bioinformatics 28: 2078-2079.

Song C.M., Lim S.J. \& Tong J.C. 2009. Recent advances in computer-aided drug design. Brief. Bioinform. 10: 579-591.

Stahelin R.V. 2014. Could the Ebola virus matrix protein VP40 be a drug target? Expert Opin. Ther. Targets 18: 115-120.

Tamilvanan T. \& Hopper W. 2013. High-throughput virtual screening and docking studies of matrix protein vp40 of Ebola virus. Bioinformation 9: 286-292.

Vinutha T., Bansal N., Prashat G.R., Krishnav V., Kumari S., Dahuja A., Sachdev A. \& Rai R.D. 2015. Three dimensional structure prediction and in silico functional analysis of $\gamma$ tocopherol methyl transferase from Glycine max. Int. J. Bioinformatics Res. 6: 289-299.

Wang J., Manicassamy B., Caffrey M. \& Rong L. 2011. Characterization of the receptor-binding domain of Ebola glycoprotein in viral entry. Virol. Sin. 26: 156-170.
Warren G.L., Andrews C.W., Capelli A.M., Clarke B., LaLonde J., Lambert M.H., Lindvall M., Nevins N., Semus S.F., Senger S., Tedesco G., Wall I.D., Woolven J.M., Peishoff C.E. \& Head M.S. 2006. A critical assessment of docking programs and scoring functions. J. Med. Chem. 49: 5912-5931.

Watanabe S., Noda T. \& Kawaoka Y. 2006. Functional mapping of the nucleoprotein of Ebola virus functional mapping of the nucleoprotein of Ebola virus. J. Virol. 80: 3743-3751.

Watt A., Moukambi F., Banadyga L., Groseth A., Callison J., Herwig A., Ebihara H., Feldmann H. \& Hoenen T. 2014. A novel life cycle modeling system for Ebola virus shows a genome length-dependent role of VP24 in virus infectivity. J. Virol. 88: 10511-24.

Wilson J., Bray M., Bakken R. \& Hart M.K. 2001. Vaccine potential of Ebola virus VP24, VP30, VP35, and VP40 proteins. Virology 286: 384-390.

Wiwanitkit V. 2014. Binding site prediction within Ebola virus VP40 protein: clue for further drug development. Asian Pac. J. Trop. Biomed. 4: 846.

Zhang Z., Li Y., Lin B., Schroeder M. \& Huang B. 2011 Identification of cavities on protein surface using multiple computational approaches for drug binding site prediction. Bioinformatics 27: 2083-2088.

Received October 7, 2016

Accepted January 26, 2017 